


\title{
La empresa inteligente ante el colapso del año 2020
}

\author{
Miguel Castro Sánchez ${ }^{1}$ y Luz María Galán Briseño \\ ${ }^{1}$ Universidad de Guadalajara, Centro Universitario de la Ciénega, m.castro@academicos.udg.mx, Av. \\ Universidad No. 1115 Colonia Linda Vista Ocotlán, Jalisco, México C. P. 47820,3929400977 \\ ${ }^{2}$ Universidad de Guadalajara, Centro Universitario de la Ciénega, luz.galan@academicos.udg.mx, Av. \\ Universidad No. 1115 Colonia Linda Vista Ocotlán, Jalisco, México C.P. 47820, 3929400974
}

Información del artículo revisado por pares

Fecha de aceptación: junio-2021

Fecha de publicación en línea: diciembre-2021

DOI: https://doi.org/10.29105/vtga7.1-104

\section{Resumen}

El año 2020 se convirtió en una oportunidad para que las empresas pudieran valorar sus estructuras y la eficiencia en sus procesos de toma de decisiones. También este año le permitió a las grandes empresas, verificar si sus planes de acción fueron preparados para cambios bruscos en el entorno. La presente investigación tiene como propósito contextualizar las consecuencias económicas ocurridas en las empresas durante el año en cuestión y distinguir a las empresas inteligentes, aquellas que lograron superar los desafíos que se les presentaron y que siguen creciendo, ya que cuentan con estructuras sólidas y planes estructurados. En consecuencia, la investigación que se realizó fue teórica y descriptiva. La fundamentación del trabajo se basó en las siguientes fuentes: comunicados emitidos por dependencias de gobierno, publicaciones realizadas por revistas especializadas, artículos científicos y libros que consideran temáticas alusivas a las empresas inteligentes. En la parte final del trabajo se concluye que la pieza clave de toda empresa inteligente es el Gerente, Director General o CEO, el cual debe aplicar su habilidad para encontrar oportunidades donde otros visualizan problemas.

Palabras clave: Empresas, líder, oportunidades.

\section{INTRODUCCIÓN}

El año 2020 es sin duda uno de los más grandes retos a los que se ha enfrentado la

\begin{abstract}
The year 2020 became an opportunity for companies to assess their structures and efficiency in their decision-making processes. Also this year it allowed large companies to verify if their action plans were prepared for sudden changes in the environment. The purpose of this research is to contextualize the economic consequences that occurred in companies during the year in question and to distinguish smart companies, those that managed to overcome the challenges that were presented to them and that continue to grow, since they have solid structures and structured plans. Consequently, the research that was carried out was theoretical and descriptive. The foundation of the work was based on the following sources: statements issued by government agencies, publications by specialized magazines, scientific articles and books that consider topics related to smart companies. In the final part of the work, it is concluded that the key piece of every intelligent company is the Manager, General Director or CEO, who must apply his ability to find opportunities where others see problems.
\end{abstract}

Keywords: Companies, leader, opportunities. JEL Codes: M14, M21.

sociedad, además de las problemáticas cotidianas a las que se enfrentaban todos los días, surgió una variante más y ahora en 
términos de salud.

Lo que se creía podía ser combatido y frenado sin problema, encontró otras variantes para ir avanzando en la vida de todas las personas y las empresas. Fue necesario hacer uso de fortalezas y recursos que se tenían en reserva para situaciones contingentes, pues dicha contingencia se hizo presente.

Inicia 2021 y aunque los efectos se valoren como menores, sigue existiendo el cierre de empresas y la pérdida de empleos, incluso los efectos económicos continúan aún más devastadores, pues son producto de la acumulación de daños poco significativos en las estructuras financieras que finalmente provocaron el colapso.

Son muchas las instituciones que se han preocupado por medir las afectaciones económicas que se han padecido durante el 2020 e incluso antes, a finales del año 2019. Para mostrar las afectaciones en un contexto global, a continuación se proporciona información oficial emitida por la Organización de las Naciones Unidas durante el año 2020 al respecto:

- Información derivada del análisis de la Conferencia de las Naciones Unidas para el Comercio y el Desarrollo (UNCTAD) muestra los siguientes datos ocurridos en la primera mitad del año 2020: la inversión extranjera directa global se desplomó 49\%, de forma específica se tuvo caídas en Norteamérica del 56\%, África 28\%, Latinoamérica $25 \%$. Europa también mantuvo cifras negativas de inversión y las economías en desarrollo disminuyeron sus inversiones en un 16\%. (ONU México, 28 de Octubre del 2020).

- El producto bruto en la economía mundial tuvo una caída del $4.3 \%$ en el año 2020 según estimaciones de la UNCTAD, de forma específica se observó una caída del $5.8 \%$ en países desarrollados y $2.1 \%$ en países en vías de desarrollo (ONU México, 22 de Septiembre del 2020).
- Olivier De Schutter, experto independiente de la ONU sobre temas de pobreza extrema, destacó que si se toma coma base una línea de pobreza de 3.20 dólares, la recesión económica asociada a la COVID-19 provocaría que 176 millones de personas a nivel global sean afectadas por la pobreza, un dato que llama la atención sobre todo en tiempos de paz y tomando como referencia más importante la Gran Depresión. (ONU México, 14 de Septiembre del 2020).

Son tres los elementos que se pueden distinguir en la información emitida por la Organización de las Naciones Unidas, en primer término la pérdida de inversión extranjera asociada a la falta de confianza por parte de los dueños de capital o, en un caso extremo, la ausencia de recursos para llevar a cabo inversiones. En un segundo término, caídas muy significativas en el Producto Interno Bruto, que provocan la falta de atención de necesidades prioritarias en los países que fueron afectados y la disminución de la capacidad de compra de los habitantes. Finalmente y no menos preocupante, la consecuencia lógica de poca inversión y caída en el PIB, incremento sustancial en la cantidad de personas bajo condiciones de pobreza en el mundo, lo cual provoca un freno importante en la búsqueda de oportunidades de desarrollo.

En el panorama nacional y con respecto a las consecuencias económicas observadas en el año 2020, las cifras de igual forma no resultan alentadoras, basta observar la información que incluye el Instituto Nacional de Estadística Geografía e Informática (INEGI) en su comunicado de prensa número 617/20 del 2 de diciembre del 2020 y que corresponde al tercer trimestre de ese mismo año (INEGI, 2 de Diciembre del 2020):

- Del 100\% de empresas registradas en el país que suman cerca de un millón novecientas mil, $86.6 \%$ de éstas informaron haber tenido algún tipo de afectación por la pandemia: 
disminución de ingresos, baja demanda, escasez de insumos.

- De mayo de 2019 a septiembre de 2020 se observó una disminución de unidades económicas de $8.06 \%$ de forma global, resaltando los pequeños y medianos negocios que desaparecieron en un $21.17 \%$. Por sectores económicos, la mayor proporción de cierres se observó en Comercio con $13.75 \%$, Servicios Privados no Financieros y Manufactureros con $12.85 \%$ y $8.78 \%$ respectivamente.

Con respecto a las cifras mostradas por parte del INEGI, es necesario visualizar las cantidades, lo que implica que estamos hablando de que en el periodo de referencia cerca de 153,000 empresas registradas desaparecieron.

De forma automática las afectaciones mostradas por el INEGI tienen una vinculación directa con lo informado por la ONU, pues el cierre de empresas en México colaboró en la pérdida de inversión global, caída en el PIB e incremento de la pobreza.

Retomando el punto 2 de la información publicada por el INEGI donde se menciona que de mayo del 2019 a septiembre del 2020 21.17\% de Pequeñas y Medianas Empresas (PYMES) en México desaparecieron, vale la pena distinguir algunas de las características que forman parte de dichas empresas y pudieron haber influido en su deceso económico.

Dos referentes que permiten identificar las PYMES, lo constituyen la cantidad de personal contratado en la organización y el nivel de ventas anuales (Nuño de León, 2019, p. 11), la pequeña empresa comercial tiene contratados de 11 a 30 trabajadores, mientras que la industrial y manufacturera tienen contratados hasta 50 trabajadores; en lo que respecta a sus ventas anuales ambos tipos de pequeña empresa manejan rangos de cuatro a cien millones de pesos. Por su parte la mediana empresa comercial tiene contratados al menos a 31 trabajadores y hasta 100, mientras que la industrial y manufacturera tiene contratados a un mínimo de 51; las ventas anuales en ambos casos van de 100 a 250 millones de pesos. Con la cantidad de personal contratado y el volumen de ventas que se maneja, se podría pensar en empresas ampliamente consolidadas, ésta situación dista mucho de la realidad, sobre todo en las pequeñas empresas. Ahora bien ¿Por qué en las estadísticas del INEGI "salta" el dato del cierre de PYMES para ser considerado?, por la sencilla razón de que en México en el año 2010 éste sector junto con las Micro Empresas, representaban el 99.8\% de las unidades económicas existentes, además de aportar el 52\% del PIB y concentrar al 72\% de los empleos formales (p. 12).

Es normal y hasta lógico pensar que teniendo una participación tan significativa en la economía nacional, también su afectación fue igual de significativa en el año 2020, la pregunta es $i$ Se pudo haber evitado el cierre de un número importante de esas PYMES durante el año 2020?, antes de contestar a la pregunta, observemos varias de las características-problema que tienen éste tipo de empresas.

Según Nuño de León (2019, pp. 3940) y en acuerdo con varios analistas, la PYME presenta varios problemas que por asociación distinguen sus principales características, las problemáticas las subdivide en las siguientes categorías: planificación, gestión y control. En lo que a planificación se refiere distingue: visión limitada desde el punto de vista sistémico estratégico, debilidad en su plan de negocio inicial, alto grado de informalidad, pobre estructura organizacional, carencia en planes alternativos y medidas de previsión. Con respecto a gestión, destaca la existencia de gerentes poco competentes, experiencia limitada, falta de personal de apoyo competente, inversiones fijas sin control, débil política de personal, no atención a procesos de mejora continua, poca aceptación al cambio, temor a recibir asesoría externa, deficiente gestión del riesgo y cultura empresarial pobre. En lo relativo al control, hace énfasis en el manejo inadecuado de recursos, bajo conocimiento del estado financiero de la empresa, controles internos inciertos, mala gestión de fondos y fallas graves en materia de seguridad. 
Del conjunto de problemascaracterísticas, destacan varias en lo particular que sin duda detonan la existencia de otras relacionadas: la informalidad en su estructura, la debilidad en el perfil del administrador responsable, falta de visión estratégica, resistencia al cambio y la carencia de planes alternativos de acción.

El supuesto aplicable al tema sería el siguiente: si las PYMES hubieran contado con un líder capacitado que les permitiera fortalecer su estructura, que hubiera estado atento a lo que sucede en su entorno, interesado en renovar los procesos internos de trabajo y preocupado por estructurar los planes alternativos de acción ¿Se hubiera evitado el colapso de un número importante de éstas organizaciones?, seguramente sí, pues se habrían distinguido como organizaciones inteligentes.

Damos paso ahora a la fundamentación teórica del tema, que precisamente tiene que ver con la conceptualización y caracterización de las organizaciones inteligentes.

\section{MARCO TEÓRICO}

Iniciemos con la consideración básica de una empresa, la cual es resultado de la organización de distintos insumos (humanos, materiales, técnicos y financieros) que permite dar origen a un producto o servicio que logrará satisfacer las necesidades de un grupo específico de la población.

La consideración anterior nos permite identificar de inmediato un gran número de entidades económicas que cumplen con dicho perfil, desde aquellas que tienen un fin puramente lucrativo, hasta aquellas que miden su beneficio de acuerdo al grado en que logran mejorar la calidad de vida de sus usuarios. En ese sentido surge una pregunta ¿Dichas organizaciones están preparadas para hacer frente a condiciones adversas que se presentan en el medio ambiente?, ¿Tienen capacidad para transformar su propósito inicial y reinventarse ante nuevas condiciones del entorno?, ¿Se pueden catalogar como empresas inteligentes?, la respuesta positiva a las interrogantes anteriores, hace pensar que efectivamente son organizaciones que van más allá de una consideración básica de empresa, pues están atentas a los cambios que ocurren en su entorno. Como lo mencionan Chávez \& Torres (2012, p. 104) "La empresa que aprende es una organización inteligente".

La experiencia diaria que acumulan las organizaciones, así como ocurre con las personas, las debe llevar a ser mejores en el futuro, pues buscan continuamente la perfección como parte de su proceso de trabajo.

Para saber si una empresa se distingue como inteligente es necesario precisar varios elementos relacionados.

\subsection{Definición.}

La empresa inteligente es aquella organización que posee características distintivas poco comunes, es una empresa abierta al aprendizaje continuo, pues siempre estará incorporando elementos que le permitan mejorar sus actividades. Montoya et al. (2010, p. 16) presentan una adaptación del modelo de los cuatro pilares de la empresa inteligente, donde distinguen elementos como la gestión del conocimiento, la inteligencia social, el uso de TIC y la inteligencia colectiva.

El pilar de gestión del conocimiento aporta cantidad y calidad de conocimientos, los cuales deben surgir de las propias capacidades que tiene la organización y atender de forma suficiente sus necesidades; es un proceso de intercambio continuo donde fortalece su conocimiento con la interacción de elementos del ambiente externo que le brindan oportunidades de crecimiento. El pilar de inteligencia social, promueve el compromiso que tiene la organización con su entorno, pues se debe a las personas y comunidad que la rodean; la retroalimentación continua le permite hacer los ajustes pertinentes en sus procesos de trabajo y en el momento exacto en que se requieren, si la empresa pierde de vista a su mercado y sus integrantes, está poniendo en riesgo su permanencia y desarrollo futuro. El pilar de inteligencia colectiva descubre un elemento primordial en la organización y que se asocia al esfuerzo colaborativo que siempre se debe tener presente, los esfuerzos del líder corporativo no tendrían efecto si no se cuenta 
con el trabajo y talento de sus colaboradores y a su vez los colaboradores, ambos deben visualizarse de forma holística, pues en ese mismo sentido existe la organización. El cuarto pilar de las organizaciones inteligentes lo representan las Tecnologías de la Información y la Comunicación (TIC) pues sin ellas las empresas no podrían aprovechar los beneficios que les aporta el desarrollo tecnológico en cualquiera de sus funciones clave, el uso de plataformas digitales asociadas a las TIC rompe con las barreras del tiempo y espacio, permitiendo a la organización aspirar a metas más ambiciosas. La integralidad de estos cuatro pilares, permitirá a una organización visualizarse como inteligente, donde la mejora continua y la implementación de procesos innovadores será una actividad cotidiana.

A modo de complemento, para Lanzas et al. (2005, p. 152) las empresas inteligentes buscan en todo momento potencializar su funcionamiento, aprovechando todos los elementos asociados a sus procesos de trabajo y dando importancia al enfoque sistémico en la toma de decisiones. Bajo la perspectiva sistémica y en correspondencia a lo planteado por Montoya et al. (2010), todos los procesos de trabajo que realiza una empresa deben mantener un orden y establecer unidades de medida y tiempo que les permitan evaluar de forma continua sus resultados, en la postura de empresa inteligente siempre se debe estar monitoreando el resultado de una decisión que se toma, la racionalidad económica y estructurada debe predominar.

\subsection{Características.}

La empresa inteligente cuenta con una serie de distintivos que permiten identificarla y valorarla. Bassat (2011, pp. 45-79) distingue que las organizaciones inteligentes del giro comercial priorizan los valores humanos, él distingue un total de 37 valores, entre éstos destacan los siguientes: "amabilidad, deseo de aprender, ejemplaridad, empatía, entusiasmo, honradez, humildad, justicia, lealtad, proactividad, responsabilidad, tenacidad". En términos generales $y$ de acuerdo a la percepción del autor, se resalta el compromiso que deben tener todos los integrantes de la organización con los propósitos de la empresa y el deseo de brindar satisfacción a los clientes internos y externos mediante el firme compromiso con las acciones responsables. La propuesta del autor es congruente con lo planteado por Montoya et al. (2010) y de forma particular en el rubro que hace alusión a la inteligencia social, pues la retroalimentación continua es lo que permite que las decisiones de la empresa siempre estén cerca de los requerimientos del mercado.

Por otro lado Guns (1996, p. 23) citado en Valencillos \& Quintero (2007) plantea como principios fundamentales de la organización inteligente el trabajo colectivo y la canalización de esfuerzos individuales en beneficio de toda la organización. Siendo congruente con las aportaciones de Montoya et al. (2010), se prioriza la inteligencia colectiva. Lo cierto es que ninguna organización que busca el uso óptimo de recursos dejaría de lado la realización de esfuerzos conjuntos por parte de sus integrantes. Es necesario puntualizar que un esfuerzo individual muy sobresaliente debe detonar potencialidades en otros integrantes de la organización para que la "sinergia" se haga presente, de lo contrario dicho esfuerzo individual solo atraerá reconocimiento para una sola persona, mas no para la organización en su conjunto.

En la búsqueda de una visión integradora de los rasgos distintivos de las organizaciones inteligentes, Lanzas et al. (2005, pp. 152-154) distinguen los siguientes elementos como parte fundamental de la creación de empresas inteligentes: dominio personal, modelos mentales, visión compartida y aprendizaje en equipo. El dominio personal involucra el fortalecimiento de características personales en la búsqueda del éxito empresarial, entre las que destacan visión sólida, interés por el logro, capacidad de planear y organizar, interés por el trabajo en equipo, aceptación del riesgo y ser tolerante ante el fracaso. Los modelos mentales son visualizados como facilitares del desarrollo empresarial y que permiten la construcción de esquemas que darán origen a las alternativas de solución de problemas. La visión compartida, asocia el esfuerzo organizado que 
fortalece la generación de ideas individuales y el aprendizaje en equipo se fundamenta en la inclusión de individuos que aportan algo distinto a lo que actualmente se tiene. Lo señalado por Lanzas et al. (2005) concuerda con los planteamientos anteriores que se incluyen en éste apartado, quienes coinciden ampliamente en el uso de capacidades individuales para la integración de una visión conjunta, el término sinergia nuevamente es valorado de forma importante.

En concordancia con los autores citados anteriormente, las organizaciones inteligentes priorizan los aportes de procesos de planeación, están atentas a lo que sucede en su entorno, priorizan la atención de necesidades sociales y valoran el esfuerzo conjunto, sin perder de vista las aportaciones individuales cotidianas.

\subsection{Procesos de innovación.}

El pilar de gestión del conocimiento señalado por Montoya et al. (2010) forzosamente está asociado a los procesos de innovación en las operaciones de la empresa y que son consecuencia lógica de la realización de procesos creativos, en dichos procesos se fomenta el surgimiento de ideas novedosas que se ponen en práctica para la atención de necesidades de la comunidad. El proceso de innovación puede tener distintos enfoques: se puede asociar a la introducción de un bien o servicio a un nuevo mercado o relacionarse con la adopción de un nuevo método de trabajo en cualquiera de las áreas funcionales de la empresa.

Las organizaciones inteligentes continuamente hacen uso de la innovación para atender nuevas demandas del mercado y solucionar problemáticas internas que se podrían llegar a presentar.

El grado de inversión que una empresa dirige hacia tareas de innovación muestra una relación directamente proporcional con los beneficios que se obtienen de ésta. Mientras hay empresas que integran dentro del departamento productivo la función de innovación, existen otras que independizan la función en forma de departamento de innovación en complemento con la función de investigación y desarrollo.
Existen distintos modelos que impulsan la innovación, Escorsa \& Valls (2003 pp. 26-30) distingue los siguientes: lineal, Marquis, London Business School y Kline.

El modelo lineal asocia distintas etapas de forma consecutiva, el proceso empieza con investigación básica, avanza hacia la investigación aplicada, genera un desarrollo tecnológico y concluye con el proceso de marketing por el lanzamiento al mercado; aún y cuando se muestra un proceso ordenado, el modelo puede ser criticado al iniciar con investigación básica y no considerando las necesidades del mercado como parte del proceso de marketing. Cada una de las fases del proceso generan resultados y mejoras, de forma particular la investigación básica resulta en descubrimiento y fortalece el conocimiento científico; la investigación aplicada resulta en invenciones y permite el registro de patentes; el desarrollo tecnológico aporta información asociada al conocimiento científico; una vez que se cuenta con la innovación se fortalecen las plantas productivas, equipos y productos existentes. El éxito en la difusión y en la comercialización de las innovaciones, permite que el ciclo se reinicie de forma continua.

Por su parte el modelo de Marquis tiene como punto de partida el reconocimiento de una demanda potencial en conjunto con la factibilidad técnica, dando origen a una idea novedosa que mediante el énfasis en procesos de investigación plantea una solución a una necesidad específica, la idea se transforma en un bien tangible $\mathrm{y}$ se procede a su socialización. En éste modelo la necesidad del mercado y las posibilidades de la empresa son las que detonan el proceso innovador. La coincidencia con respecto al modelo lineal es que el proceso concluye con la socialización de la innovación mediante esfuerzos de marketing, la mayor diferencia con respecto al modelo lineal, es que se parte de los requerimientos del mercado, lo que asegura una mejor aceptación de la innovación; la aplicación de ésta modalidad del proceso, sin duda destaca a las organizaciones inteligentes.

El modelo London Business School prioriza el ejercicio del liderazgo efectivo, la 
disponibilidad de recursos y la utilización de sistemas y herramientas para el incremento de la productividad. En su fase crítica, integra las funciones de innovación de procesos y adquisición de tecnología para la generación de nuevos conceptos y desarrollo de productos.

El modelo de Kline considera las necesidades del mercado potencial y pasa por varias etapas de diseño: analítico, detallado y prueba, rediseño y producción; concluye con la comercialización de la invención. En éste modelo existe una retroalimentación constante con procesos de investigación que permiten hacer los ajustes correspondientes. En congruencia con el Modelo Marquis, se inicia con los requerimientos de mercado, pero se confiere mayor importancia al cumplimiento de varias etapas en el diseño y la retroalimentación con procesos de investigación.

Además de resaltar la importancia que tienen los modelos de innovación en el ejercicio de las organizaciones inteligentes también es necesario visualizar el enfoque hacia el que una empresa se quiere acercar. En todo momento el modelo de innovación seleccionado debe estar debidamente estructurado y partir de las demandas que muestra el mercado y las posibilidades que tiene la empresa de satisfacerlas; la inclusión de procesos investigación, diseño y registro legal también son tareas ineludibles y, como parte final y no menos importante, las innovaciones deben socializarse y comercializarse mediante el trabajo que realizará el área de marketing. Es importante mencionar que de nada sirve innovar si no se parte de una necesidad del mercado, pues de dicha satisfacción surgen los recursos para seguir impulsando los procesos creativos y de innovación.

\subsection{Las TIC en la toma de decisiones.}

El uso de las técnicas de información y comunicación sin duda han provocado un cambio significativo en la forma como operan las empresas inteligentes, los software aplicables a las actividades cotidianas en la empresa han influido en una mayor eficiencia en el alcance de objetivos corporativos. En opinión de Gándara et al. (2007) las TIC han hecho más eficiente el proceso de toma de decisiones en los siguientes aspectos: reducen el tiempo de respuesta, proporcionan suficiente información, reducen los niveles de incertidumbre y permiten la identificación de distintos objetivos importantes para la organización.

El gran potencial de las TIC ha permitido que pequeñas empresas incursionen en el terreno de la innovación disruptiva, convirtiéndose en entes corporativos en muy corto tiempo, las TIC permiten aumentar la intensidad de una idea de negocio y su cobertura. La ejemplificación que se hará más delante de organizaciones inteligentes permite visualizarlas como producto de las TIC, gracias a las plataformas virtuales en las cuales tienen establecidos sus negocios.

\subsection{Valoración del riesgo y la incertidumbre.}

Una organización inteligente es aquella que no teme al riesgo y es capaz de visualizar una oportunidad en él. El riesgo obliga a cambiar y no conformarse, a ser mejores antes de que un competidor se lo exija. La total certeza en la toma de decisiones en el contexto actual es una falacia, es necesario generar variantes en las actividades que realiza una empresa, evitar ser absorbidos por la zona de confort y estar abiertas a la experimentación de negocios nuevos.

El riesgo en una característica permanente de las empresas actuales. La habilidad del líder de la empresa inteligente debe proveerle de la información suficiente para enfrentar los riesgos de todo tipo de situaciones. De forma similar al riesgo, una decisión incierta puede esconder una oportunidad o un peligro, pero la habilidad del líder y la retroalimentación constante permitirán especificar el terreno que se está explorando. Como lo mencionan Martínez et al. (2017, p. 134) "la incertidumbre puede producir desconcierto y desánimo...pues lo que ayer hacíamos hoy quizá no sirve".

$\mathrm{La}$ incertidumbre exige a las organizaciones inteligentes estar alertas y no confiarse de un éxito obtenido, el cambio continuo en el entorno obliga a la mejora en 
los procesos de trabajo y la mayor eficiencia en el uso de recursos.

\section{MÉTODO}

Como se mencionó en el apartado de resumen y la introducción, el trabajo desarrollado constituyó una investigación de tipo documental y descriptiva. El punto de partida fue el problema que vivieron las empresas a raíz de la pandemia COVID-19 y la influencia que tuvo en su desarrollo económico. Posteriormente y como una alternativa para aminorar los efectos de la problemática, se desarrollaron los fundamentos teóricos asociados al tema empresas inteligentes, para distinguir las características que deben tener las organizaciones para no verse afectadas de sobre manera por contingencias similares a las vividas en el año 2020. Los fundamentos teóricos fueron recuperados de artículos científicos, publicaciones de instituciones de reconocido prestigio, en forma de libros, artículos o boletines de prensa.

En un siguiente apartado se incorporaron evidencias de empresas inteligentes a criterio de consultoras y/o publicaciones de prestigio, donde la innovación y el uso de TIC eran elementos que las distinguían. Finalmente se resaltaron las características de las organizaciones inteligentes y se enfatizó la necesidad de que las PYMES atendieran dichas características para fortalecer sus cimientos organizacionales y evitar afectaciones desastrosas derivadas de cambios en su medio ambiente.

\section{RESULTADOS}

Como producto de los análisis previos es momento de identificar las empresas, que a criterio de organizaciones de prestigio, puedan catalogarse como inteligentes y que distingan entre sus características lo referido por Montoya et al. (2020): gestión del conocimiento, inteligencia social, TIC e inteligencia colectiva y que sean congruentes con las cualidades derivadas del análisis de Nuño de León (2019): líderes capacitados con visión estratégica y organizaciones con una estructura formal sólida, adaptabilidad al cambio y con planes alternativos debidamente estructurados.

Con la intención de encontrar un referente que nos permita destacar ejemplos de organizaciones inteligentes y cuáles son sus características, nos dimos a la tarea de hacer una investigación en distintas fuentes de información asociadas al tema, los referentes eran muy concretos con respecto a la estructura de las empresas: líderes en innovación, generadoras de conocimiento, estructuras organizaciones bien cimentadas, líderes capacitados, promoción de esfuerzos conjuntos en sus colaboradores y atentos a las necesidades del mercado.

Como resultado de la investigación de referentes, fue posible ubicar publicaciones de diversas organizaciones consultoras vinculadas a instituciones educativas $\mathrm{o}$ agrupaciones de expertos disciplinares de reconocido prestigio, que se han dado a la tarea de valorar y distinguir a las empresas que han sido capaces de sobresalir en su sector y que incluso se han posicionado en los primeros lugares en el contexto mundial; una de éstas organizaciones es la Agencia Boston Consulting Group (BCG) que generó un ranking en el año 2020 de las empresas con mejores prácticas en términos de tecnología innovadora (Entrepreneur staff, 25 de Junio del 2020). Según el listado, las 10 empresas mejor ubicadas y de acuerdo a su grado de importancia fueron: Apple, Alphabet, Amazon, Microsoft, Samsung, Huawei, Alibaba, IBM, Sony y Facebook.

La razón de utilizar éstos referentes fue verificar si los fundamentos manejados por distintos autores en la caracterización de las empresas inteligentes se hacen presentes y ver la posibilidad de que otras organizaciones usen ésta información para modificar los elementos de su estructura y poder hacer frente a situaciones contextuales como las ocurridas en el año 2020. La realidad es que varias de las empresas citadas se mantuvieron vigentes en el año 2020 e incluso vieron mejorados sus resultados, en particular aquellas que fueron proveedores de bienes y servicios a través de plataformas virtuales.

La pregunta que surge en éste momento es: ¿Cuáles son las características que distinguen a éstas organizaciones inteligentes?, en primer término poseen una estructura claramente definida que tiene al 
frente un director general o CIO altamente capacitado; utilizan de forma continua las tecnologías de información y comunicación (TIC) pues como se mencionó anteriormente, varias de ellas tienen su principal operación en una plataforma virtual específica; la innovación es una herramienta que utilizan de forma cotidiana, pues incluso la promoción que hacen de sus productos se centra en sus procesos continuos de mejora y en la excelente forma de cubrir las necesidades de sus clientes; existe un alto compromiso individual de sus trabajadores con su rendimiento y con la aportación que hacen a la misión de la empresa y la realización de trabajo colaborativo; finalmente $y$ no menos importante, están atentos a los cambios que ocurren en el entorno y en particular, las nuevas exigencias de su mercado. Precisando los criterios marcados por Montoya et al. (2020) y Nuño de León (2019), sin duda pueden clasificarse como empresas inteligentes.

Las empresas distinguidas por la BCG en algún momento fueron PYMES y lograron mejorar sus procesos a tal grado que hoy sus decisiones influyen de forma significativa en un número importante de personas, sean trabajadores, inversionistas o consumidores. Dichas empresas iniciaron sus operaciones mucha antes del año 2020 y seguramente seguirán vigentes mucho tiempo después de ese año, para ellos las experiencias vividas en ese año son un aprendizaje que les permitirá seguir mejorando sus procesos de trabajo, ese es precisamente uno de los distintivos de las empresas inteligentes: el aprendizaje continuo.

Las PYMES existentes deben tratar de emular a las grandes empresas de acuerdo a sus posibilidades. Cualquier empresa desde sus inicios se debe preocupar por prácticas que la lleven a fortalecer su estructura a futuro, haciendo una analogía con el proceso de construcción de una casa, las micro y pequeñas empresas, son los cimientos de las medianas y grandes empresas que se integrarán a futuro, si los cimientos son débiles la estructura final tendrá esa misma característica.

En opinión de Valecillos \& Quintero (2007) y en directa alusión a las PYMES, la incorporación de novedades en lo que a dirección de empresas se refiere, contribuye al fortalecimiento de los procesos de toma de decisiones, entre las novedades aplicables se encuentra: el cuadro de mando integral, los sistemas de justo a tiempo, inteligencia emocional, análisis financieros y de marketing, etc. La incorporación de los elementos anteriores permitiría fortalecer la función de dirección y el fortalecimiento de la estructura operativa de la organización.

\section{CONCLUSIONES}

El problema de salud que se está enfrentando desde finales del año 2019 y que sigue presente hasta nuestros días, ha provocado que las empresas hagan transformaciones muy importantes en sus bases de operación, de manera particular las ha obligado a fortalecer: su estructura organizacional, la capacitación de los responsables de administrarlas, escuchar de forma atenta a sus clientes (internos y externos), priorizar los procesos de innovación y el uso de las TIC. Las ha llevado a la integración de planes de acción alternativos, a valorar de mejor forma a sus colaboradores y preocuparse por su capacitación y desarrollo profesional, incluso ha provocado que se atiendan de mejor forma las políticas de bioseguridad.

Los ejemplos de empresas inteligentes que se incluyen como referencias, deben ser una aspiración de las pequeñas y medianas empresas sobre su constitución ideal, pues lo que resulta cierto y contundente, es que varias de esas grandes empresas se vieron fortalecidas por los acontecimientos ocurridos en el año 2020, en particular las asociadas a la venta de productos o servicios por medio de plataformas virtuales y las que brindaban herramientas para la comunicación virtual.

El director general o CIO de la empresa juega un papel fundamental, pues debe ser el guía en las funciones claves de la misma: cambios en el medio ambiente, nuevas exigencias de los clientes y uso intensivo de las TIC para fortalecer el ejercicio de la innovación.

La empresa que se retroalimenta de forma continua minimiza sus posibilidades 
de falla, pues hace los ajustes que se requieren en el momento exacto. 


\section{REFERENCIAS}

Bassat, L. (2011). Inteligencia comercial. Editorial Plataforma.

Chávez, N. \& Torres, G. (2012). La organización inteligente en un ambiente de aprendizaje: una exploración de sus aspectos generales. AD-minister. (21), 101-115. En: https://www.redalyc.org/pdf/3223/322327351007.pdf

Rubio, J. (15 de Septiembre del 2015). El método de Mark Zuckerberg: así se trabaja en Facebook. El País. https://verne.elpais.com/verne/2015/09/15/articulo/1442316243_513348.html

Entrepreneur staff. (25 de Junio del 2020). Empresas más innovadoras del mundo. Entrepreneur. https://www.entrepreneur.com/article/352373

Escorsa C. P. \& Valls, P. J. (2003). Tecnología e innovación en la empresa. Ediciones UPC. En: $\mathrm{https} / / /$ books.google.es/books?hl=es\&lr=\&id=vFZsgeizTO8C\&oi=fnd\&pg=PA7\&dq=inno vacion\&ots $=$ H7G4yjaZ41\&sig=_kt9WXm-

k1SUfCQP_uZqF8hwluY\#v=onepage\&q=innovacion\&f=false

Gándara, V. J. A., Mathison, B. L. E., Primera, L. C. A. \& García, G. L. R. (2007). Efectos de las TIC en las nuevas estructuras organizativas: de la gerencia vertical a la empresa horizontal. Revista NEGOTIUM. 3 (8), 4-29. En: https://www.redalyc.org/pdf/782/78230802.pdf

INEGI. (2 de Diciembre del 2020). El INEGI presenta resultados de la segunda edición del ECOVIDIE y del estudio sobre la demografía de los negocios 2020. Comunicados de prensa. https://inegi.org.mx/contenidos/saladeprensa/boletines/2020/OtrTemEcon/ECOVIDIE_DEMOGNEG.pdf

Lanzas, A. M., Lanzas, V. E. \& Arias, M. L. (2005). Creación de empresas inteligentes, cinco disciplinas que contribuyen al proceso. Scientia et Technica. XI (28), 151-154. En: https://www.redalyc.org/pdf/849/84911707027.pdf

Martínez, G. M., Gómez, P. H. \& Martínez, G. J. A. (2017). La gestión de la incertidumbre: empresas inteligentes con trabajadores del conocimiento. Redipe. 6(8), 132-143. En: https://revista.redipe.org/index.php/1/article/view/299

Montoya, A., Mejía, A. \& Bravo, M. (2010). Sistemas avanzados de gestión. Organizaciones inteligentes. Universidad de San Buenaventura Seccional Cali. En: file:///C:/Users/brise/Downloads/Sistemas_avanzados\%20_gestion_organizaciones_intelige ntes.pdfNuño de León, M. R. (2019). Administración de pequeñas empresas. Red Tercer Milenio.

En: http://www.aliat.org.mx/BibliotecasDigitales/Hospitalidad/Administracion_de_pequenas_e mpresas.pdf

ONU México. (28 de Octubre del 2020). La inversión extranjera cae la mitad por la pandemia de COVID-19. Información oficial de las Naciones Unidas. https://coronavirus.onu.org.mx/lainversion-extranjera-cae-a-la-mitad-por-la-pandemia-de-covid-19

ONU México. (22 de Septiembre del 2020). UNCTAD advierte sobre la posibilidad de una segunda recesión "de una magnitud irreconocible”. Información oficial de las Naciones Unidas. https://coronavirus.onu.org.mx/unctad-advierte-sobre-la-posibilidad-de-una-segundarecesion-de-una-magnitud-irreconocible

ONU México. (14 de Septiembre del 2020). La recesión económica causada por la pandemia de la COVID-19 puede provocar que 176 millones de personas caigan en la pobreza, alerta experto de la ONU. Información oficial de las Naciones Unidas. https://coronavirus.onu.org.mx/la-recesion-economica-causada-por-la-pandemia-de-lacovid-19-puede-provocar-que-176-millones-de-personas-caigan-en-la-pobreza-alertaexperto-de-la-onu

Valencillos, C. \& Quintero, N. (2007). Enfoque de las organizaciones inteligentes en la implementación de nuevas técnicas de dirección en las pequeñas y medianas empresas (PYMEs). Revista de Ciencias Sociales. 13(2), 278-289. En: https://www.redalyc.org/pdf/280/28011677007.pdf 